\title{
A HREM study of a nearly perfect $\Sigma=11$ tilt bicrystal in nickel
}

\author{
Danièle Bouchet $\left({ }^{1}\right)$ and Jany Thibault $\left({ }^{2}\right)$ \\ (1) Laboratoire de Physique des Solides, U.A C.N.R.S. 002, Bât. 510, UPS, F91405 Orsay Cedex, \\ France \\ (2) DRFMC/Service de Physique des Matériaux et Microstructure, CENG, 85X, F38041 Grenoble \\ Cedex, France
}

(Received January 24, 1992; accepted March 31, 1992)

\begin{abstract}
Résumé. - Un bicristal de nickel contenant un joint de grains $\Sigma=11$ (311) de flexion autour de [011] est étudié par microscopie électronique à haute résolution. D’après les résultats d'analyse Auger et d'attaques chimiques, la queue du bicristal contient beaucoup plus de soufre que la tête. La structure du joint de grains pris en tête et en queue a été examinée en MEHR. Les parties parfaites symétriques ont été comparees à la structure calculée par minimisation d'énergie. Le joint contient des dislocations dont la distribution est différente en tête et en queue de bicristal. La relation entre impuretés ségrégées et structure du joint est discutée.
\end{abstract}

\begin{abstract}
A nickel bicrystal containing a (311) [011] $\Sigma=11$ grain boundary is investigated by high resolution electron microscopy. From Auger analysis and chemical etchings, the end of this bicrystal is known to contain more intergranular sulfur than the head. The structure of the GB extracted from the head and the end of the bicrystal is studied by HREM. The perfect symmetrical part $\Sigma=11$ will be compared to the structure computed by energy minimization. The GB contains GB dislocations whose distribution is different at the head and in the end of the bicrystal. The presence of impurities related to the GB structure is investigated.
\end{abstract}

\section{Introduction.}

In this paper, we present a high resolution electron microscopy (HREM) study of a nickel tilt bicrystal $\Sigma=11$ whose misorientation angle is $50.48^{\circ}$ around the common tilt axis [011] and whose plane is (311) common to both grains. The general frame of this work is the relationship between the intergranular structure and segregation of impurities and its consequences on the engineering properties of materials.

Investigations of the atomic structure by HREM of tilt grain boundaries exist in the literature. They have been carried out in gold [1,2], molybdenum [3] and aluminium [4]. Since the authors in [1] used tilted beam illumination their results are questionable. The problem encountered in these experimental conditions is that the positions of the intensity maxima shift laterally as the defocus and the thickness vary. 
Some preliminary TEM studies at mean resolution, of polycrystalline nickel $[5,6]$ and $\Sigma=11$ nickel bicrystal [7] suggested that the inclination of the grain boundary plane and the deviation from the exact coincidence position was much more important for the intergranular segregation ability of the grain boundary than the value of the index coincidence $\Sigma$. The authors experimentally showed that sulfur preferentially segregated in the grain boundaries (GB) whose interface plane was the less compact. As shown in [8], the GB density is geometrically related to the interplanar distance, even in the case of asymmetrical GBs.

On the other hand, the structure of the perfect $\Sigma=11 \mathrm{~GB}$ has been first investigated by computer simulations in aluminium $[9,10]$. More recently, the energy and the structure of the $\Sigma=11 \mathrm{~GB}$ in Ni have been computed [11]. Furthermore, the segregation energies of s-p valence impurities have been investigated in [11] for the two possible symmetrical planes (311) and (332) of this $\Sigma=11$ GB. The calculations use the tight-binding type electronic theory and the results suggest that the energy segregation is much lower for the (311) plane which is the second densest plane in fcc materials.

Conventional TEM study gives only access to the mean GB plane of the interface: at that level, it is difficult to detect the microfacetting of the GB. The aim of our work is to better characterize the structure of the GB by using HREM.

\section{Experimental.}

\subsection{THE TILT BICRYSTAL $\Sigma=11(311)\left(50^{\circ} 48\right.$ AROUND $\left.<011>\right)$.}

2.1.1 Fabrication and composition. - The nickel bicrystal, fixed at the Ecole Nationale Supérieure des Mines de Saint Etienne has been grown by solidification along the $<011>$ direction starting from a sced with a (311) GB plane (Fig. 1a).

The bicrystal contains less than $10 \mathrm{ppm}$ wt of sulfur; the global composition is given in the figure $1 \mathrm{~b}$; the bulk composition is almost constant from the head to the end of the bicrystal.

2.1.2 Characteristics of the $G B$ and samples preparation. - Auger experiments and chemical etching performed on different areas of the bicrystal revealed the existence of an intergranular sulfur concentration gradient starting from the bicrystal head (for further details see [7]).

In the following, two different thin foils will be investigated: the first one is extracted from the head and the other one from the end of the bicrystal (Fig. 1a). Three millimeters diameter carots around the grain boundary are made and then sliced in about $80 \mu \mathrm{m}$ thick discs which are then thinned by double jet electropolishing.

2.2 HREM OBSERVATION CONDITIONS. - High resolution observations were performed on a JEOL $4000 \mathrm{EX}$ microscope running at $400 \mathrm{kV}$. The spherical aberration coefficient $C_{\mathrm{s}}$ is $1 \mathrm{~mm}$, the beam divergence is about $1 \mathrm{mrd}$ and the defocus spread is $8 \mathrm{~nm}$.

The common $<011>$ tilt axis of the bicrystal is positionned paralled to the electron beam in such a way that the GB plane and the (111) planes are viewed end-on together (Fig. 2a). The images were made using an objective aperture including the 13 following beams: the transmitted beam, the 111 beams (belonging to both grains) whose corresponding interplanar spacing is $d_{111}=$ $2.03 \AA$ and the 200 beams from both grains too (Fig. $2 \mathrm{~b}$ ). 


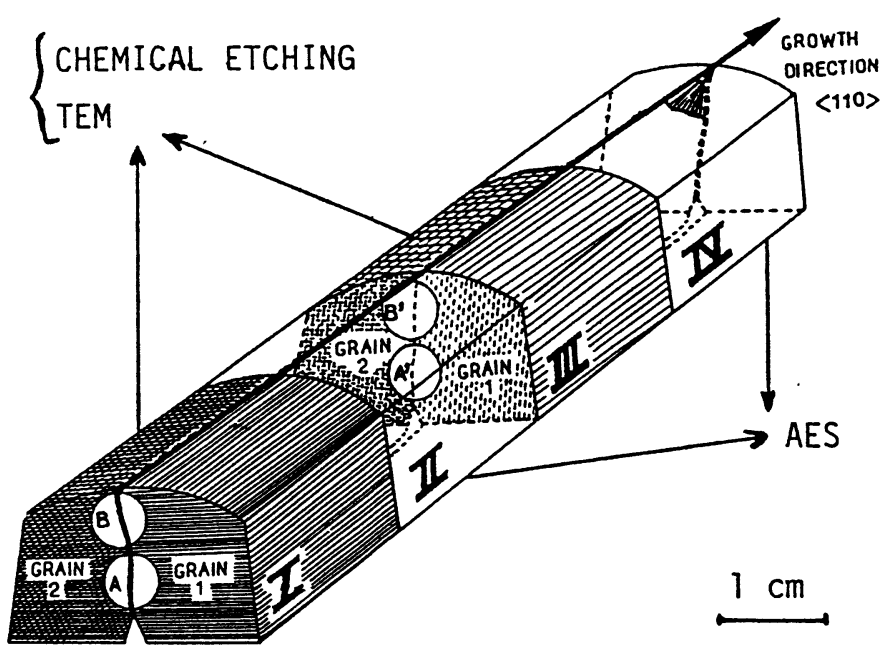

a

\begin{tabular}{|l|c|c|c|c|c|c|c|c|c|c|c|c|}
\hline & $\mathrm{O}$ & $\mathrm{S}$ & $\mathrm{Al}$ & $\mathrm{Fe}$ & $\mathrm{Ca}$ & $\mathrm{As}$ & $\mathrm{Sb}$ & $\mathrm{V}$ & $\mathrm{Cu}$ & $\mathrm{Pb}$ & $\mathrm{Si}$ & $\mathrm{P}$ \\
\hline PART I & $<20$ & $<10$ & 190 & 235 & 15 & 4 & $<1$ & $<1$ & 11 & 2 & 50 & $<20$ \\
\hline PART III & $<20$ & $<10$ & 190 & 230 & $<10$ & 5 & $<1$ & $<1$ & 11 & $<1$ & 20 & $<20$ \\
\hline
\end{tabular}

b

Fig. 1. - Description of the $\Sigma=11$ nickel bicrystal: a) schematic representation; two thin foils are investigated: one is extracted from the head (part I) and the other one from the end (part III) of the bicrystal. b) Bulk composition of two parts of the bicrystal in weight ppm.

\section{Results.}

3.1 COMPARISON BETWEEN THE HEAD AND THE END OF THE BICRYSTAL - Large views of the GB recorded at the head and at the end of the bicrystal are shown in figure 3 . They exhibit clear geometrical differences.

At the head of the bicrystal (Fig. 3a), the grain boundary looks almost perfect. It is symmetrical with respect to the (311) GB plane and the misorientation between the two grains is about $50^{\circ}$. It is slightly smaller than the exact coincidence position. One can notice the presence of a few defects (associated with GB steps) along this grain boundary.

At the end of the bicrystal (Fig. 3b), the GB becomes clearly asymmetrical: the grain boundary plane is no longer (311). The interface is no more rectilinear but facetted and waved. The misorientation angle is about $48.5^{\circ}$ : the deviation from exact coincidence position is smaller by about 2 degrees. Numerous intergranular defects are present along this interface. The analysis of these defects will be done in the following. 


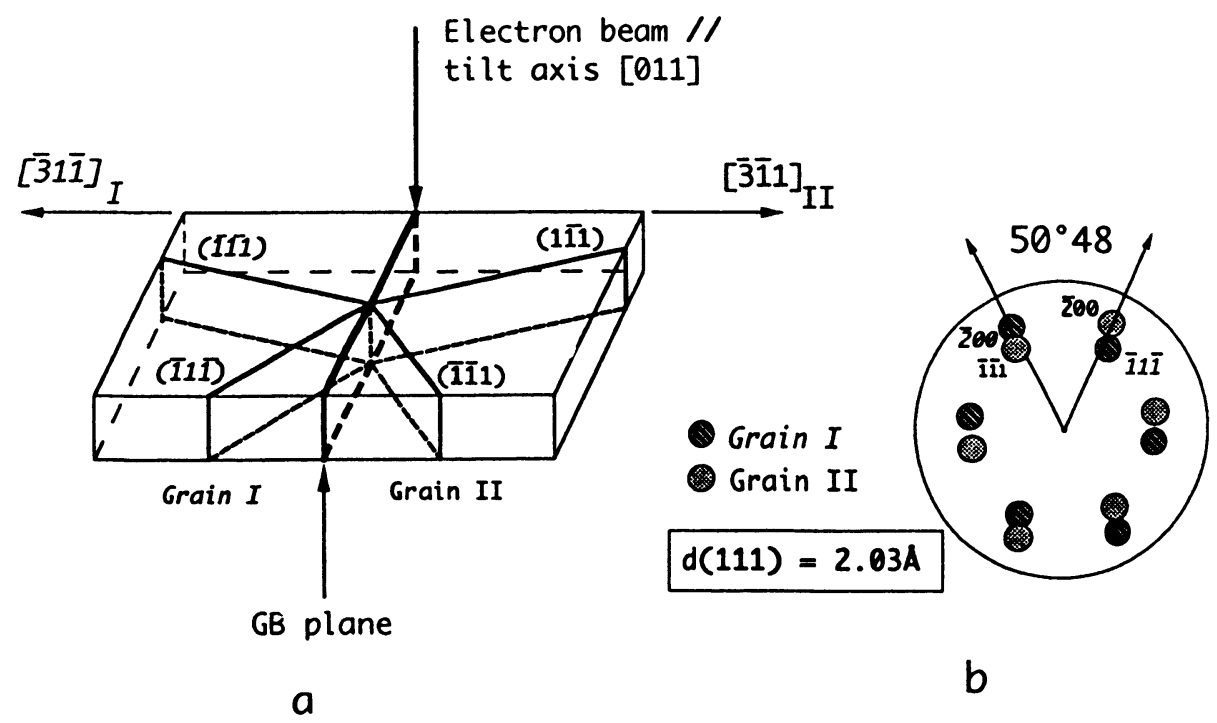

Fig. 2. - HREM observation conditions (JEOL 4000EX, $C_{\mathrm{s}}=1 \mathrm{~mm}$ ) : a) specimen geometry: the GB plane and the 111 planes are viewed end-on together, the tilt axis is parallel to the incident electron beam. b) the transmitted beam and the 111 and 200 beams are selected by the objective aperture to make the image.

3.2 STRUCTURE OF THE PERFECT $\Sigma=11$ GRAIN BOUNDARY. - To undergo the HREM study of the defects along the interface, the structure of the perfect $\Sigma=11$ boundary needs to be described in a precise manner in order to estimate the deviation from a perfect reference structure. One can describe the GB structure either geometrically or/and energetically. We will present now both approaches.

3.2.1 GB geometry: CSL and DSC description (Fig. 4). - The geometrical approach of a perfect GB is based on the coincidence site lattice (CSL) description. The CSL corresponds to the coincidence of the two adjacent lattices. By definition, the $\Sigma$ value is the ratio between the volume of the primitive cell of CSL and the volume of the primitive crystal cell. A geometrical description of the $\Sigma=11 \mathrm{~GB}$ has been given in detail in [12]. The primitive CSL lattice is based on the three vectors:

$$
\begin{aligned}
& A=a / 2[\overline{1} 2 \overline{1}]_{\mathrm{I}}=a / 2[12 \overline{1}]_{\mathrm{II}} \\
& B=a / 2[1 \overline{1} 2]_{\mathrm{I}}=a / 2[\overline{1} \overline{1} 2]_{\mathrm{II}} \\
& C=a[31 \overline{1}]_{\mathrm{I}}=a[3 \overline{1} 1]_{\mathrm{II}}
\end{aligned}
$$

The displacement shift complete lattice (DSC) has been introduced [13] in order to describe a small deviation from the exact coincidence position. This DSC lattice is the set of the linear combinations between the vectors of the two lattices. As a consequence, a defect with a Burgers vector belonging to the DSC lattice leaves the GB structure invariant. A small deviation from the coincidence is thus compensated by a periodic distribution of grain boundary dislocations (GBDs) whose Burgers vectors are DSC vectors. In the case of $\Sigma=11$, the DSC lattice is based on the three vectors:

$$
\begin{aligned}
& a=a / 22[\overline{174}]_{\mathrm{I}}=a / 22[174]_{\mathrm{II}} \\
& b=a / 22[147]_{\mathrm{I}}=a / 22[\overline{147}]_{\mathrm{II}} \\
& c=a / 11[31 \overline{1}]_{\mathrm{I}}=a / 11[3 \overline{1} 1]_{\mathrm{II}}
\end{aligned}
$$


Head of the bicrystal

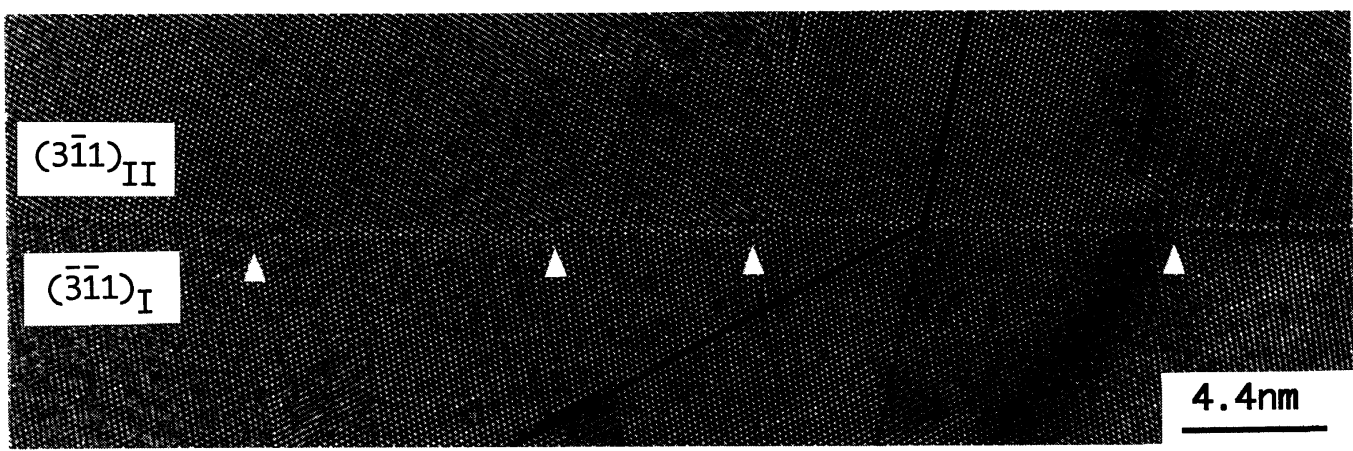

$50^{\circ}$

\section{End of the bicrystal}

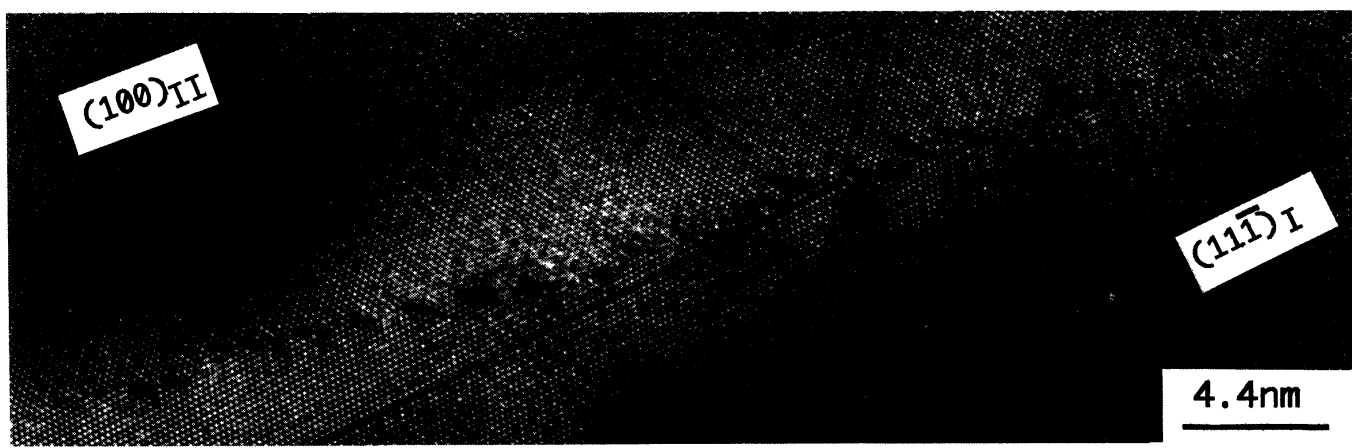

$48^{\circ} 5$

Fig. 3. - HREM overview of the head and of the end of the $\Sigma=11$ grain boundary. It has to be noticed that the tilt angle and the inclination of grain boundary plane vary from the head to the end. Theoretically, the exact coincidence misorientation angle is $50^{\circ} 48$ and the perfect GB plane is (311). At the head of the bicrystal, large facets of the GB are (311) symmetrical plane with a small number of defects. At the end of the bicrystal the GB is asymmetrical, the density of defects is larger than in the head.

Figure 4a shows the CSL and the DSC lattices projected along the [011] common axis. The trace of the GB plane lies along the direction $[\overline{2} 3 \overline{3}]_{\mathrm{I}}$ common to $[23 \overline{3}]_{\mathrm{II}}$. The following smallest DSC vectors are described in figure $4 \mathrm{~b}$ :

$b_{\mathrm{g}}=a / 22[\overline{2} 3 \overline{3}]_{\mathrm{I}}=a / 22[23 \overline{3}]_{\mathrm{II}}$ which is parallel to the GB plane, and whose length is $b_{\mathrm{g}}=0.75 \AA$. This vector is obtained by the linear integer combination: $a-b$.

$b_{\mathrm{c}}=a / 11[31 \overline{1}]_{\mathrm{I}}=a / 11[3 \overline{1} 1]_{\mathrm{II}}$ which is perpendicular to the GB plane and with $b_{\mathrm{c}}=1.06 \AA$

$b_{\mathrm{x}}=a / 22[\overline{174}]_{\mathrm{I}}=a / 22[174]_{\mathrm{II}}$ which is parallel to the GB plane and with $b_{\mathrm{x}}=1.30 \AA$

$b_{\mathrm{d}}=a / 22[45 \overline{5}]_{\mathrm{I}}=a / 22[81 \overline{1}]_{\mathrm{II}}$ which is the sum of the two first DSC vectors $b_{\mathrm{c}}$ and $b_{\mathrm{g}}$. Its length is $1.30 \AA$ the same as the $b_{\mathrm{x}}$ one.

$b_{\mathrm{y}}=a / 22[283]_{\mathrm{I}}=a / 22[465]_{\mathrm{II}}$ whose length is $b_{\mathrm{y}}=1.4 \AA$.

Each GBD is associated with a geometrically necessary GB step based on the CSL-DSC geometry and whose characterization will be shown in section 3.3.1. 


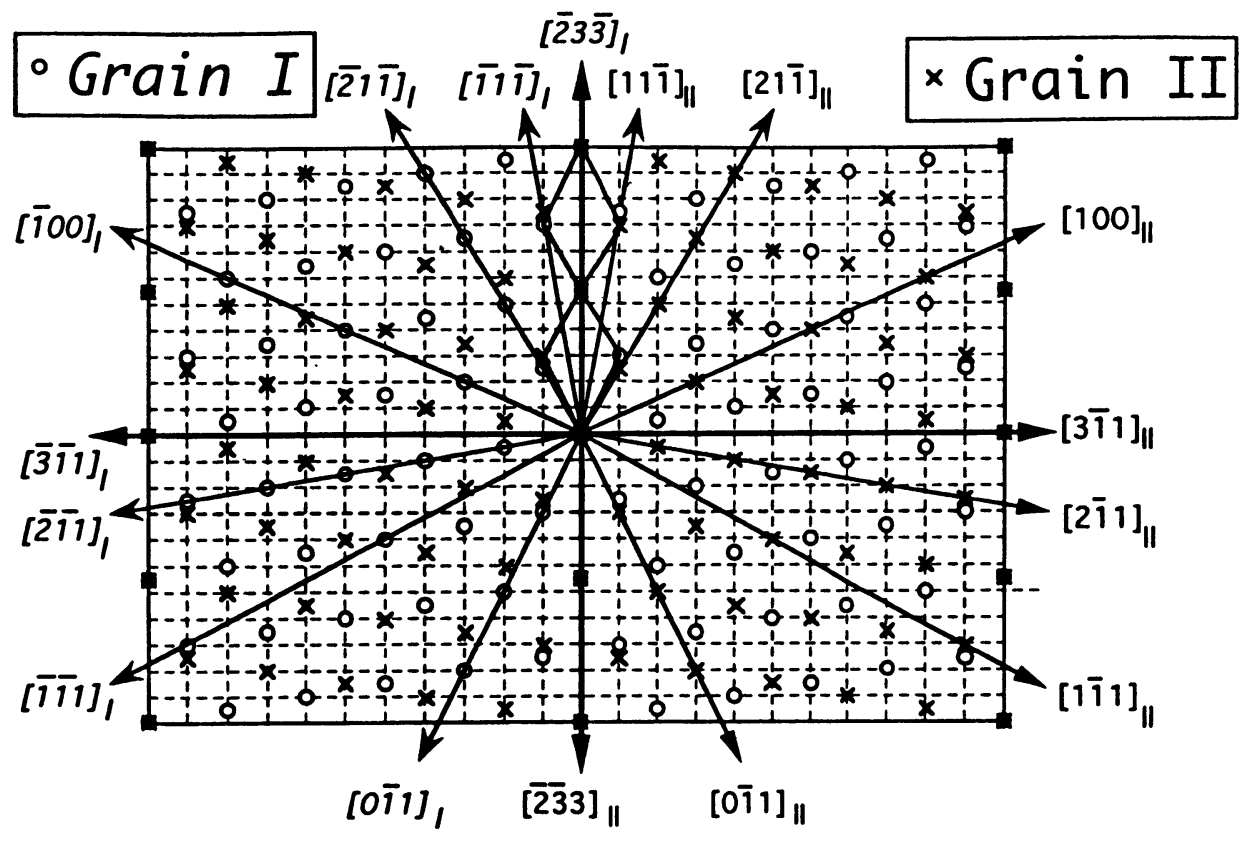

a

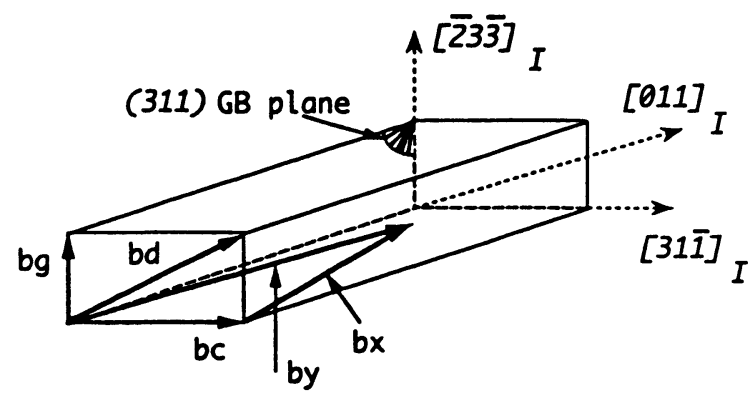

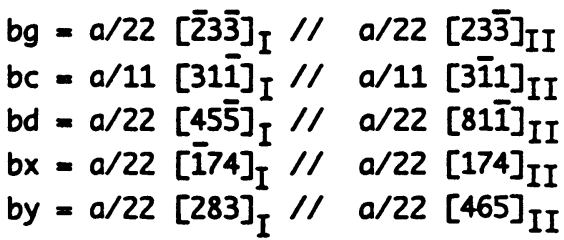

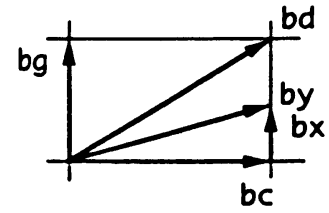

$l=0.75 \AA$

$l=1.06 \AA$

$l=1.30 \mathrm{~A}$

$l=1.30 \mathrm{~A}$

$l=1.40 \mathrm{~A}$

Fig. 4. - Geometrical description of the perfect symmetrical grain boundary [011] (311) $\Sigma=11:$ a) four [011] projected cells of the coincidence site lattice (CSL): the projected DSC lattice is superimposed on the CSL. One GB period with two identical structure units is drawn. b) DSC lattice geometry showing the DSC vectors used in the paper. The axes are referred to the grain $I$. 
3.2.2 Calculated relaxed structure and HREM image simulation of the perfect GB. - In the following, we shall use the lowest energy configuration obtained in [11] and we shall compare it to the experimental images. The electronic potential calculated from this theoretical structure is shown on the top left of figure 5. It does not present any rigid body translation: the (311) GB plane is a pure mirror. This GB is a favoured GB and is made up with only one type of structural unit [14].

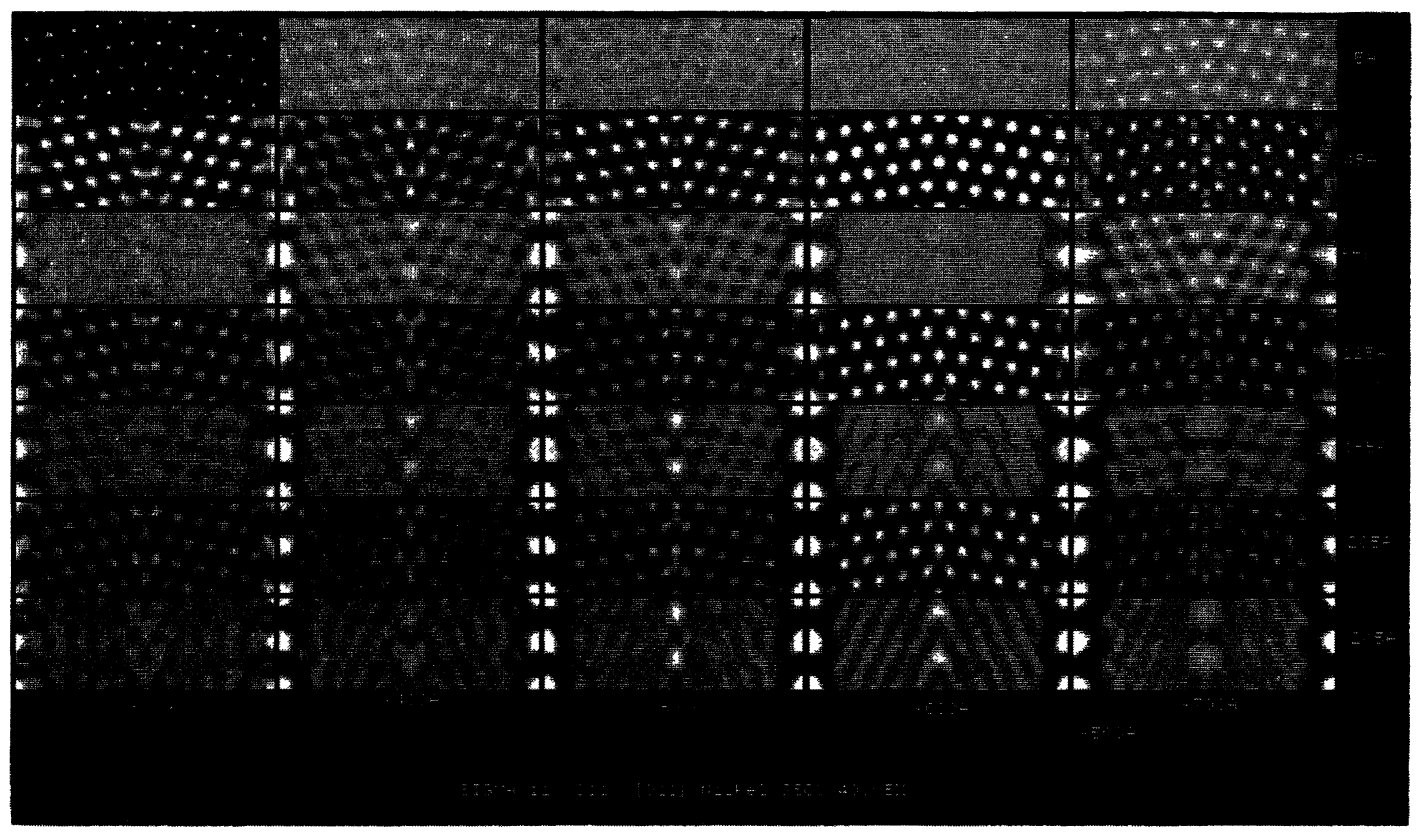

Fig. 5. - Structural model and HREM simulation. On the top left: simulated potential from the structure calculated by Larère et al. [11]. The other images are the HREM image simulations of the interface as a function of the foil thickness (vertical) and of the defocus (horizontal).

We used the Kilaas program [15] to simulate the HREM images. Figure 5 shows the result of the simulation of the GB images obtained with the atomic positions from [11] and the evolution of the contrast as a function of the foil thickness and the defocus. At a defocus of about $-60 \mathrm{~nm}$, the contrast of the atomic columns is maximum and positive (white atoms). Moreover, whatever the focus, it does not change very much with thickness. From an experimental point of view, this last result will facilitate the interpretation of the images in terms of atomic positions.

Figure 6 shows the comparison (white atoms) between the experimental HREM image of the boundary extracted from the head of the bicrystal where the GB looks almost perfect and the simulated image of the perfect symmetrical (311) $\Sigma=11 \mathrm{~GB}$. From the comparison, it might be concluded that the GB experimental structure is in very good agreement with the structure proposed in [11]. Unfortunately, we are not able to present the images in the focusing conditions giving the reversed contrast and this might leave a small doubt on the exact structure. However, the structure is so simple that we will use it in the following as the starting point. 


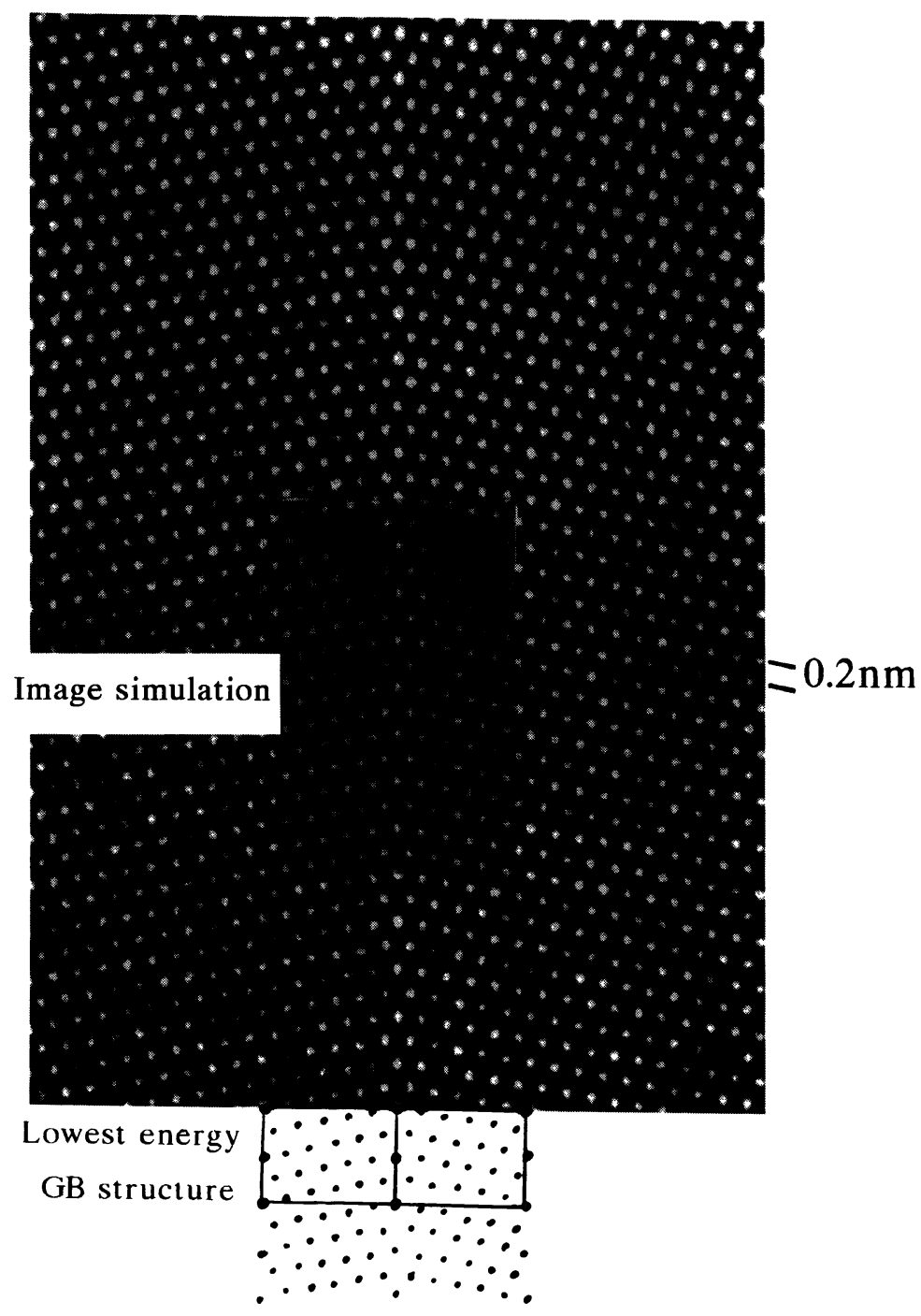

Fig. 6. - Comparison between the simulated and the experimental image of the perfect $\Sigma=11$ grain boundary.

\subsection{INTERGRANULAR DEFECTS STUDY.}

3.3.1 Characterization of the GB defects: Burgers vector determination. - The technique used to determine the projected Burgers vectors of the secondary dislocations and the associated step height has been previously described [16]. This determination procedure uses the CSL and DSC lattices. Starting from the coincidence lattices defined on each side of the defect, the displacement vectors are determined in the two grains as $s_{1}$ and $s_{2}$. The Burgers vector of the dislocation is thus given by:

$$
\mathbf{b}=\mathbf{s}_{1}-\mathbf{s}_{2}
$$


and the associated step is:

$$
h=\left(\mathbf{s}_{1}+\mathbf{s}_{2}\right) \cdot \mathbf{n} / 2
$$

where $\mathbf{n}$ is the normal to the GB plane from grain I to grain II.

Our sign conventions are given elsewhere [17]. In the following, the step height will be refered to the length $h_{0}$ of the $b_{\mathrm{c}}$ vector $\left(b_{\mathrm{c}}=a / \sqrt{\mathbf{1 1}}=1.06 \AA\right.$ in Ni).

3.3.2 Defects characterization at the head of the bicrystal (Fig. 7). - As it has been seen in figure 3, the grain boundary is symmetrical on its main part but a network of isolated GB dislocations is superimposed; these dislocations are not perfectly equidistant, since their spacings vary from $70 \AA$ to $110 \AA$. We can consider that they are separated by a mean distance of $90 \AA$. The Frank's formula $(\Delta \theta=b / d)$ applied to the components perpendicular to the GB plane leads to a decrease of the misorientation angle of $\Delta \theta=-0.7^{\circ}$. In fact, as shown in figure 7 , the dislocation components are not all perpendicular to the GB plane and only three on them contribute to the misorientation angle; the sum of the perpendicular components $\left(2.5 b_{c}\right)$ is distributed on about $500 \AA$. Thus, on this figure, the deviation angle $\Delta \theta$ is $-0.3^{\circ}$. These results are in the same order of magnitude as the one found by conventionnal TEM on the same bicrystal [7].

Figure 7 shows the analysis of a sequence of intergranular defects in this part of the bicrystal. These GBDs are DSC dislocations, as mentionned in section 2. It has to be noticed that the associated steps are large and have not all the same sign.

One has to point out that the GBD with $2 b_{c}(h=0)$ has not been detected along this boundary although it is geometrically possible.

3.3.3 Defects characterization at the end of the bicrystal (Fig. 8). - In this part of the grain boundary, the density of the defects is much larger than in the head of the bicrystal. The defects are separated by about $20 \AA$ which leads to a deviation from exact coincidence position of $\Delta \theta=-2.5^{\circ}$. The contrast of these defects is less good than in the head of the bicrystal. Despite the apparent complexity of the image, it is possible to analyze them: one can see on figure 8 that some perfect parts of $\Sigma=11$ can be recognized and consequently used to draw Burgers circuit around a defect.

As for the bicrystal head, a sequence of defects has been studied (Fig. 8). The Burgers vectors found in this part are similar to the ones described in the previous paragraph but the steps have all the same sign. As the distance between GBDs is small, this makes the GB plane change.

One has to point out as in section 3.3.2, that the defects present in the GB are generally associated with a large step.

\section{Discussion and conclusion.}

The goal of our work was to go further in the understanding of the relationship between the presence of segregated impurities, and the structure of the GB.

Our HREM observations show that the GB structure is different at the head and at the end of the bicrystal. Beside this, other experiments revealed intergranular sulfur segregation at the end of the bicrystal [7]. These are the experimental results. However, at this stage of the discussion, our HREM or STEM [19] observations fail to detect the presence of intergranular sulfur atoms. So, in the absence of a direct proof, at the TEM level, of sulfur segregation, it remains impossible to assert that the evolution of the structure from the head to the end of the bicrystal is related to the presence of a larger amount of sulfur in the end. Let us notice that the only study revealing the presence of impurities by HREM was performed on Mo [3] where the authors discussed the influence of oxygene on the structure of the grain boundary dislocations (GBDs).

Nevertheless, some simple considerations could be emphasized from the HREM observations schematically summarized in figure 9. At a nanometer level, the GB plane is made with GBDs 


\section{Bicrystal head}
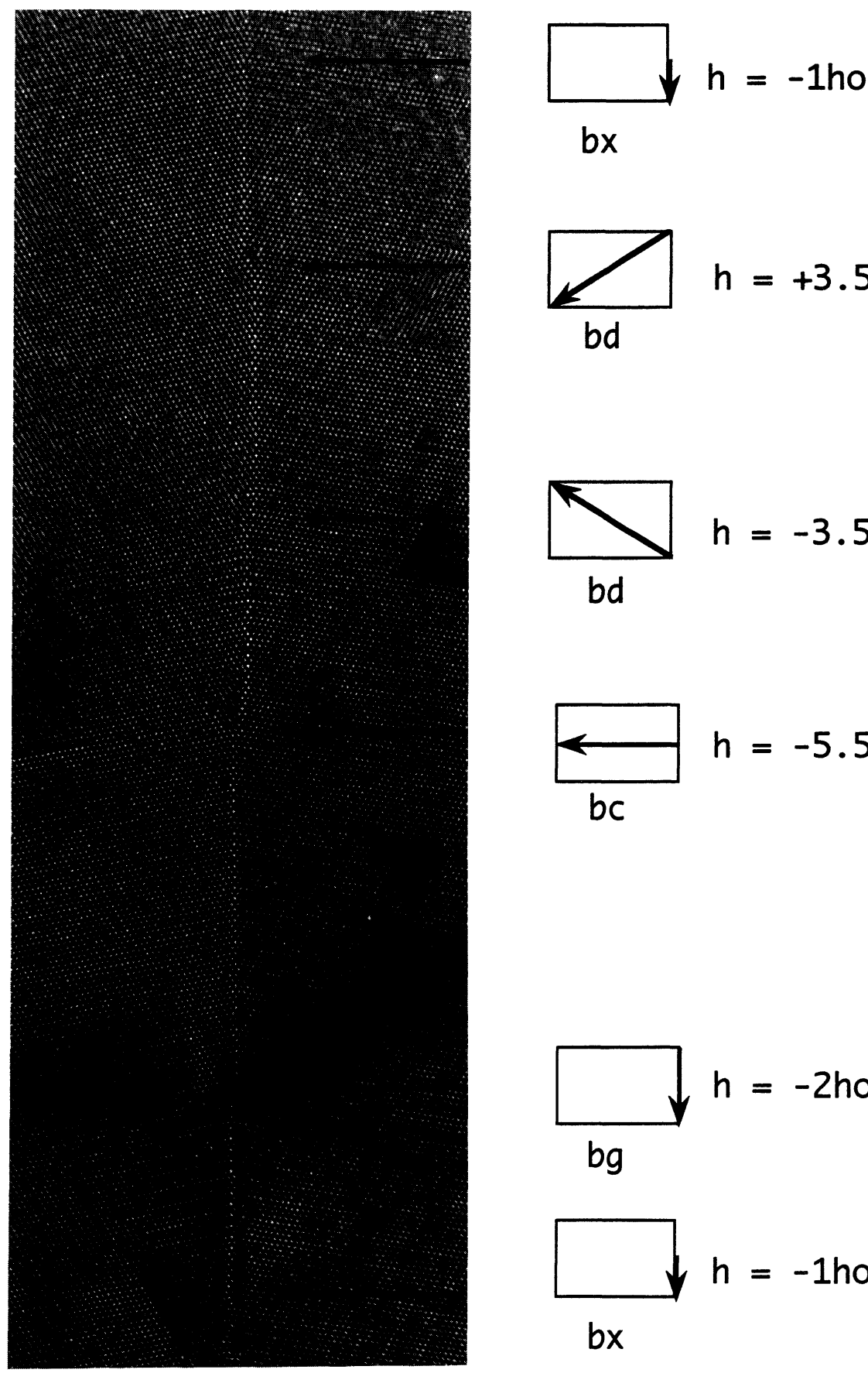

bx

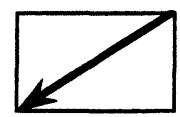

$h=+3.5 h o$

bd

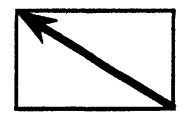

$h=-3.5 h o$

bd

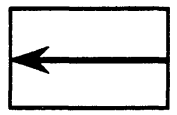

$h=-5.5 h o$

bc

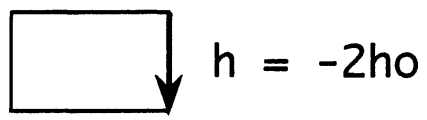

bg

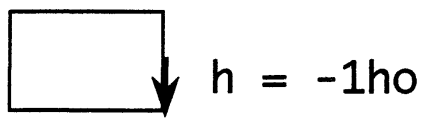

bx

Fig. 7. - Analysis of a defect sequence in the head of the bicrystal. The defects are DSC dislocations: the step height is referred to $h_{0}=b_{\mathrm{c}}=1.06 \AA$. 
Bicrystal end

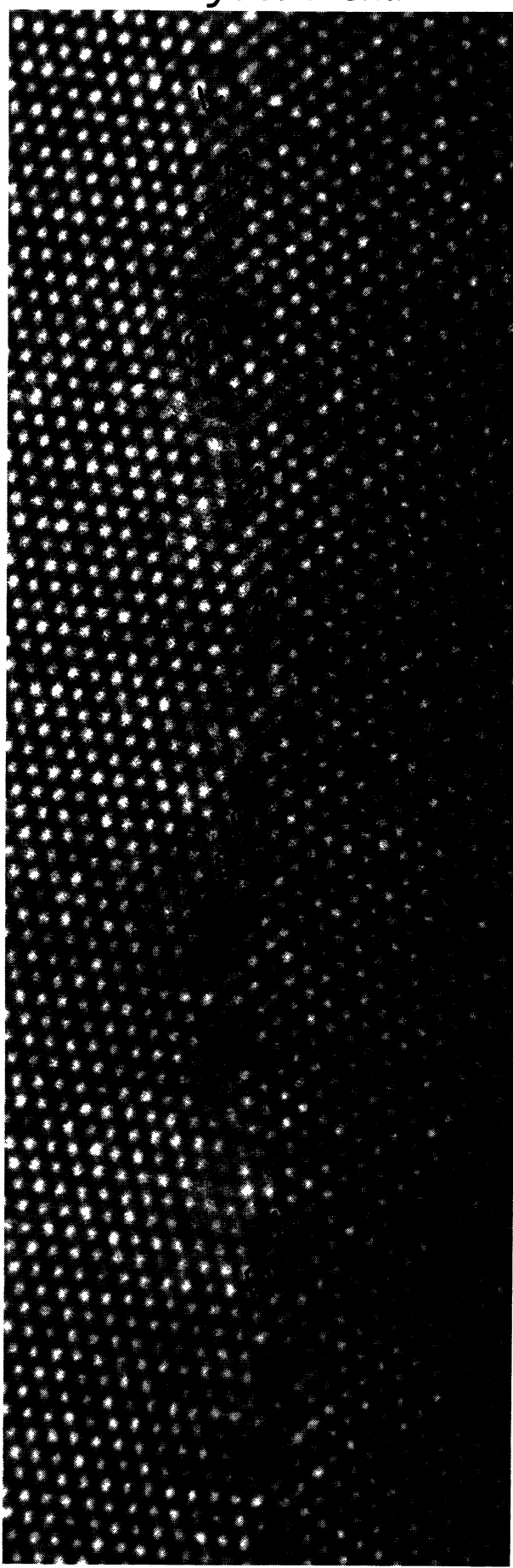

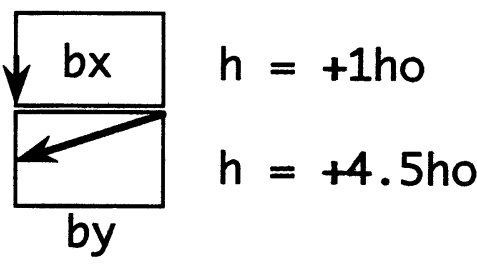

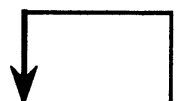

$h=+2 h o$

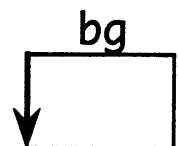

$h=+9 h o$

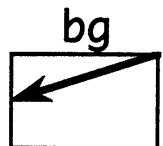

by

$h=+4.5 h o$

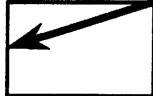

$h=+4.5 h o$

by

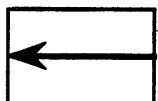

$h=+5.5 h o$

bc

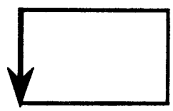

$h=+9 h o$

bg

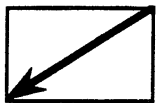

$h=+3.5 h o$

bd

Fig. 8. - Analysis of an intergranular defect sequence in the end of the bicrystal. The defects are the same DSC dislocations as in figure 7 . 
separated with perfect GB SUs (even if these SUs are locally distorted) which are known to contain almost no segregation [11]. Especially if the defects are numerous, the GB plane could no longer be viewed as a perfect homogeneous interface both from the structural point of view and from the segregation capability as well. At this atomic level, the GB plane concept fails. Therefore, it is no more possible to consider the GB plane as a structural criterion for the intergranular segregation ability, as suggested previously [5-7].

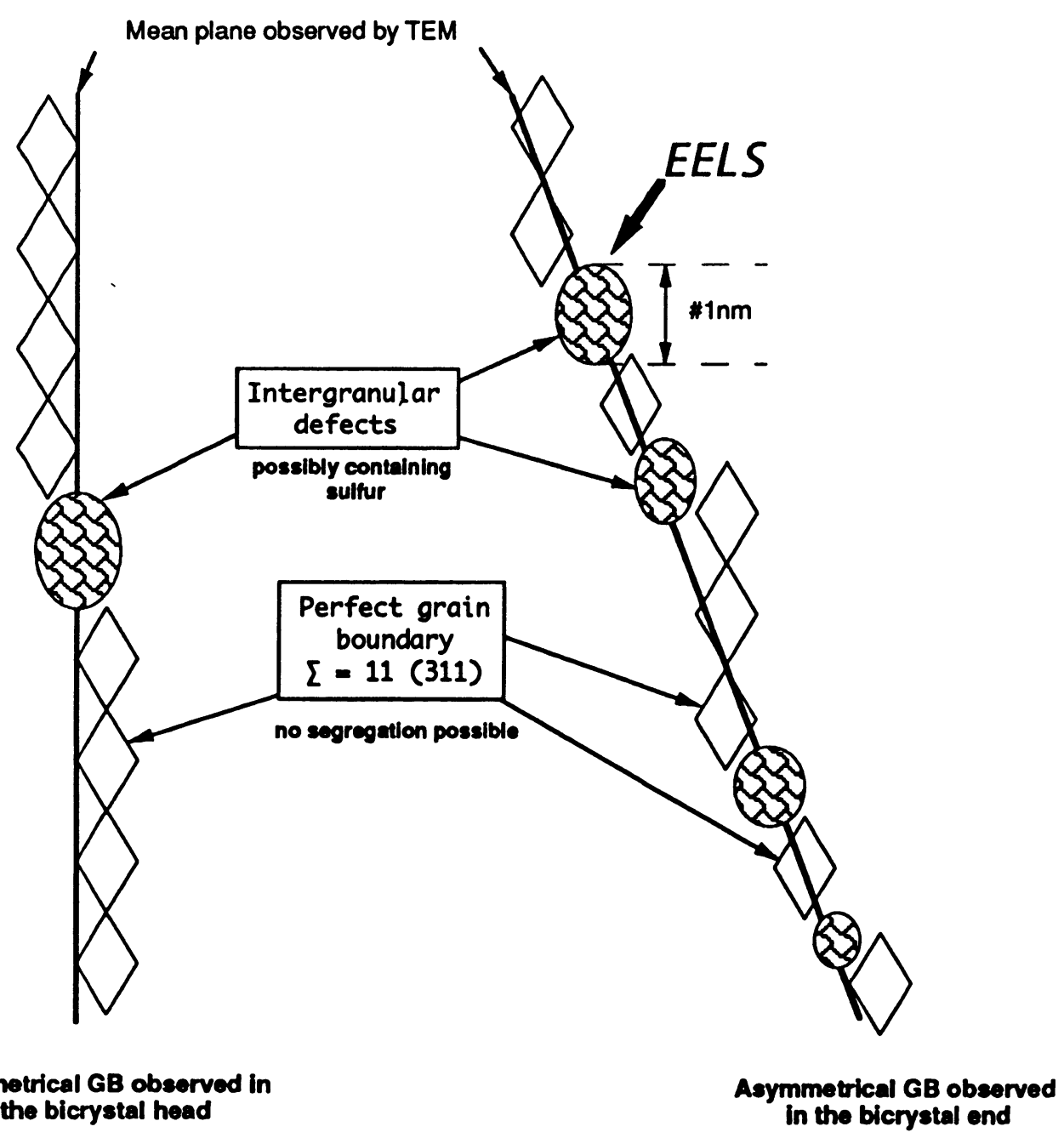

Fig. 9. - Scheme of the grain boundary plane which is composed of perfect grain boundary parts separated by defects.

Besides this point, is it possible to understand the GB structure variation along the bicrystal? At the head of the bicrystal, a small deviation from the exact coincidence position is accommodated by DSC dislocations. As a matter of fact, the smallest DSC vectors can geometrically exist with two possible opposite GB steps. Thus, the presence of GBDs with opposite steps might be the result of the energy decrease by the increase of the configurational entropy. 
The structure found at the end of the bicrystal can be described by the accumulation of GBDs with the same GB step sign. This results in a change of the GB plane and a deviation from the exact coincidence angle.

The question remains if it would be possible to obtain a symmetrical GB with a misorientation angle of $48.5^{\circ}$ by the accumulation of DSC dislocations without step or with opposite sign steps as observed [18] in elementary semiconductors. However, one has to notice that the configurations obtained in [18] had been induced by a symmetrical deformation of bicrystals.

One of the ways to explore in order to get some tendency is the simulation. The energy of all the possible configurations have to be estimated by computer simulations and the presence of segregated impurites as well in order to see if this would stabilize one of them.

\section{References}

[1] Krakow W., WetZel J.T., SMith D.A., Philos. Mag. A53 (1986) 739.

[2] ICHINOSE H., IsHIDA Y., J. Phys. Colloq. France 46 (1985) C4-39.

[3] Penisson J.M., Nowicki T.N., BisCondi M., Philos. Mag. A58 (1988) 947.

[4] Penisson J.M., Dahmen U., Milis M., Philos. Mag. Lett. 64 (1991) 277.

[5] BOUCHET D., PRIESTER L., Scripta Met. 21 (1987) 475.

[6 Bouchet D., Aufray B., Priester L., J. Phys. Colloq. France 49 (1988) C5-17.

[7] SWIATNICKI W., LARTIGUE S., BisCondi M., BouChET D., J. Phys. Colloq. France 51 (1990) C1-341.

[8] Wolf D., J. Phys. Colloq. France 46 (1985) C4-197.

[9] HASSON G., BoOs J.Y., HeRbeuval I., Biscondi M., Goux C., Surf. Sci. 1 (1972) 115.

[10] POND R.C., VITEK V., SMITH D.A., Acta Metall. 27 (1979) 235.

[11] LaRERE A., GuIlloPE M., MASUDA-Jindo K.I., J. Phys. Colloq. France 49 (1988) C5-47.

[12] RAE C.M.F., SMITH D.A., Philos. Mag. A41 (1980) 477.

[13] BollmanN W., Crystal defects and crystalline interfaces (Heidelberg, Springer Verlag, 1970).

[14] SuTtON A., VITEK V., Philos. Trans. R. Soc. London A309 (1983) 1.

[15] KILAAS, Berkeley.

[16 KING A.H., SMITH D.A., Acta Cryst. A36 (1980) 335.

[17] ElkajbajI M., Thibault-DesseauX J., Philos. Mag. A58 (1988) 325.

[18] Thibault J., PutauX J.L., Michaud H.M., Baillin X., Jacoues A., George A., Inst. Phys. Conf. 117 (1991) 111.

[19] Bouchet D., Colliex C., Flora P., Krivanek O., Mory C., Tence M., Microsc. Microanal Microstruct. 1 (1990) 443. 